linear portions covering the range $44 \cdot 47 \geqslant \varepsilon>19 \cdot 13$ (that is, $10^{2} \Delta \varepsilon^{-1}=2.9787$ ) and $12.03 \geqslant \varepsilon>4.00$ (that is, $10^{2} \Delta \varepsilon^{-1}=$ 17.6874). Even in the event of the plot being a straight line, the molecular significance of the trends in association constant with bulk permittivity are not obvious. For this reason, other correlations must be sought. One such possibility is the relation between $\log K_{A}$ and the mean molecular weight of the solvent. For relations of this sort, comparison between the properties of solutes in various solvents is facilitated by converting derived parameters, for example, association constants expressed in molarities, $K_{A}(c)$, t) corresponding thermodynamic parameters using molo fraction concentration units ${ }^{2}$, such as $K_{A}(x)$ (assuming densities at 80, 87, 91 and 95 wt per cent dioxan in water are equal). Thus this removes the cratic contributions leaving the required unitary quantities ${ }^{3}$. In Fig. 1 comparison is made between plots of $\log K_{A}$ against both $\varepsilon^{-1}$ and the mean molecular weight, $\bar{M}$, for lithium iodide in dioxan-water mixtures at $25^{\circ} \mathrm{C}$. The correlation with solvent molecular weight is at least as good as that with permittivity and is perhaps preferable since the permittivity is also some function of solvent composition. Correlation between a derived quantity, for example $K_{A}$, with a continuum function begs the question of how the latter is related to solvent composition.

Department of Chemistry,

M. J. Blandamer

University of Leicester.

Received March 10, 1967.

${ }^{1}$ Fuoss, R. M., J. Amer. Chem. Soc., 80, 5059 (1958),

${ }^{2}$ Atkinson, G., and Mori, Y., J. Chem. Physics, 45, 4716 (1966)

${ }^{3}$ Gurney, R. W., Ionic Processes in Solution (McGraw-Hill, London, 1953).

\section{A Direct Electrical Measure of Adsorption} on Electrodes

EuECTRICAx measurement of the impedance of electrodesolution interfaces involves experimental determination of the impedance of the whole electrochemical cell and correction for the ohmic drop across the internal cellresistance that is in series with the interface under study. This ohmic drop is not negligible in investigations of redox processes (alternating current polarography ${ }^{1}$ ) or of adsorption-desorption reactions (tensammetry ${ }^{1}$ ).

Correction for the resistance involves additional experiments; data obtained by calculation for the interfacial impedance consequently are of lower accuracy than can be achieved in the experimental determination of the imprdance of the cell as a whole; furthermore, the range of interfacial impedances that can be investigated has a limit (at low impedance) for any given desired precision.

It has been pointed out ${ }^{2}$ that the degree to which sinusoidal alternating signals are rectified by adsorptiondesorption processes should give a quantitative measure that requires no correction for series-resistance effects provided the cell is polarized by an alternating eurrent of controlled waveform and magnitude. We are now able to report that this prediction has been experimentally verified.

Instrumentation similar to that described for the study of redox processes under controlled alternating current ${ }^{3}$ was used; in addition, a wave-analyser was connected across the cell and rectification measured as the second harmonic component of the alternating voltage across the cell. A fixed alternating current was applied to the cell and the total voltage across the latter, as well as the second harmonic voltage, was observed. A decade resistance-box was connected in series with the cell in such a manner that it appeared as part of the cell itself (that is, measurements were made across the cell plus the adjustable resistance).

Confirmation of the earlier prediction was obtained in that variation of the resistance of the cell drastically altered the total alternating voltage across the cell but had no effect on the magnitude of the second harmonic component of the voltage.

The experiment succeeds to a degree that depends on the extent to which the applied alternating current is free from second harmonic. For example, when the applied alternating current contained 0.5 per cent second harmonic, the distortion induced by the tensammetric process (of half-saturated amyl alcohol in a molar solution of sodium sulphate) was unaffected by increased cell resistance of up to $2,000 \mathrm{ohms}$; when the harmonic content of the applied current was decreased by a factor of ten the measured distortion was unaffected by added cell resistance as high as $20,000 \mathrm{ohms}$. The cell resistance itself can normally be kept below $100 \mathrm{ohms}$, and therefore the requirements for a suitable source of alternating current are not at all stringent. The precision of the measurement of the distortion will be approximately the same as the percentage of the second harmonic in the applied signal.

All the ramifications of the new approach described here cannot be foreseen, but certain advantages are immediately apparent: for example, measurement of adsorption as a function of time at the dropping mercury electrode is a most involved procedure when interfacial impedance is used as an indicator (in fact, no such work has been published despite the valuable information that is, in principle, to be obtained) whereas display of the second harmonic voltage as a function of time offers no special difficulties.

We now intend to explore the extent to which measurements of rectification can provide information about the mechanisms, energetics, and kinetics of adsorption phenomena.

HenRy H. BaUer

A. K. Shallial

Department of Chemistry, University of Kentucky, Lexington, Kentucky.

Received March 17, 1967.

${ }^{1}$ Breyer, B., and Bauer, H. H., Alternating Current Polcrography and Tensammetry (Interscience Publishers, New York, 1963).

${ }^{2}$ Bauer, H. H., and Foo, D. C. S., Austral. J. Chem., 19, 1103 (1966).

${ }^{3}$ Baver, H. H., Britz, D., and Foo, D. C. S., J. Electroanal. Chem., 9, 481 (1965)

\section{Effect of Introducing a Sulphur Bridge on the Herbicidal Activity of Diquat}

DIQUAT ${ }^{1}$, paraquat ${ }^{2}$ and related diquaternary salts from $2,2^{\prime}$-and $4,4^{\prime}$-bipyridyls ${ }^{3}$ have attracted much attention as herbicides $^{4,5}$. Their mode of action is thought ${ }^{6-8}$ to be connected with their ability to be reduced to a stable radical cation at a potential $\left(E_{0}\right)$ of about -0.35 to $-0.45 \mathrm{~V}$, the stability of the free radical being due to the delocalization of the odd electron over both pyridine rings. The influence of the introduction between the pyridine rings of an atom or group which is capable of acting as an electron transfer bridge has not so far been investigated. This communication reports our results with diquaternary salts of $2,2^{\prime}$-dipyridyl sulphide.

Before this investigation no diquaternary salts of 2,2'-dipyridyl sulphide had been prepared. The dimethiodide of $2,2^{\prime}$-dipyridyl sulphide $\left(\mathrm{I} ; R=\mathrm{CH}_{3}, X=\mathrm{I}\right)$ was obtained by reacting $2,2^{\prime}$-dipyridyl sulphide with boiling methyl iodide for $6 \mathrm{~h}$. The solid which precipitated during the reaction consisted of a mixture of the dimethiodide and the mono-methiodide salts. When the solid was treated with cold ethanol the mono-methiodide salt dissolved. The almost pure dimethiodide which remained undissolved crystallized from aqueous ethanol as yellow crystals, m.p. $212^{\circ} \mathrm{C}$ (with decomposition). (Found: C, 30.65; H, $3 \cdot 2 ; \mathrm{N}, 5 \cdot 6 ; \mathrm{S}, 6 \cdot 9 ; \mathrm{I}^{\prime}, 53 \cdot 2 . \mathrm{C}_{12} \mathrm{H}_{14} \mathrm{I}_{2} \mathrm{~N}_{2} \mathrm{~S}$ requires $\mathrm{C}, 30 \cdot 9$; $\mathrm{H}, 3.0 ; \mathrm{N}, 5 \cdot 9 ; \mathrm{S}, 6 \cdot 8 ; \mathrm{I}^{\prime}, 53.8$ per cent.) The ultra-violet 